\title{
AN OBSERVATION PROBLEM FOR THE BESSEL DIFFERENTIAL OPERATOR
}

\author{
K.-D. WERNER ${ }^{1}$
}

(Received 14 February 1983; revised 12 May 1983)

\begin{abstract}
In this paper, the parabolic partial differential equation $u_{t}=u_{r r}+(1 / r) u_{r}-\left(\nu^{2} / r^{2}\right) u$, where $\nu \geqslant 0$ is a parameter, with Dirichlet, Neumann, and mixed boundary conditions is considered. The final state observability for such problems is investigated.
\end{abstract}

\section{Introduction}

In this paper, we consider the final state observation problem for the parabolic differential equation involving the Bessel differential operator of order $\nu=0$ or $\nu \in\left[\frac{1}{2}, 1\right]$, namely

$$
u_{t} \equiv B_{(0,1)}^{\nu} u, \quad \text { where } B_{(0,1)}^{\nu} \psi \equiv-\frac{1}{r} \frac{d}{d r}\left(r \frac{d \psi}{d r}\right)+\frac{\nu^{2}}{r^{2}} \psi \text { and } r \in(0,1) .
$$

$t$ denotes the time and $r$ the spatial variable.

We understand a process to be a solution of a partial differential equation or, as in our case, a solution of an evolution equation in a Banach space. Therefore, let $S(t)$ be a strongly continuous semigroup of bounded operators defined on a reflexive Banach space $X$ for $t \geqslant 0$. For $u_{0} \in X$ we define $u(\cdot) \in C[0, T ; X]$ by $u(t)=S(t) u_{0}$ and call it the trajectory of $u_{0}$. For such a trajectory the observation operator $C$ : $\mathscr{D}(C) \subset X \rightarrow Y$ is defined by $C u_{0}=H S(\cdot) u_{0}, u_{0} \in \mathscr{D}(C)$, where $H: X \rightarrow V$ is the observing operator. The 'final state' operator $F: X \rightarrow X$ is defined by $F u_{0}=S(T) u_{0}, u_{0} \in X$. The final state observation problem consists of the question as to whether or not $\left\|F u_{0}\right\|_{X}$ is bounded relative to $\left\|C u_{0}\right\|_{Y}$. The

\footnotetext{
' School of Mathematics, University of New South Wales, P. O. Box 1, Kensington, N.S.W. 2033, Australia. (Presently on leave from the Department of Mathematics, University of Kassel, University of the State of Hesse, 35 Kassel, West Germany.)

(c) Copyright Australian Mathematical Society 1984, Serial-fee code 0334-2700/84
} 
existence of a reconstruction operator $G: Y \rightarrow X$ such that $F=G C$ amounts to continuous constructibility of the final state from the observations $H S(\cdot) u_{0}$.

As a concrete example, consider a physical model of small vibrations (diffusion) of a gas in a cylinder or the heat transfer in a solid cylinder, where the temperature at any point depends only upon the distance of that point from the axis of the cylinder. Then, the problem consists of determining the temperature distribution $u(r, T)$ in the moment $T>0$ by measuring the temperature $u(\theta, t)$ at a fixed point $\theta \in[0,1]$ during the time 0 to $T$. Therefore, we ask whether it is possible to reconstruct the temperature distribution $u(r, T)$ at the fixed time $T>0$ if the initial condition is not known.

Dolecki [4], [5], Dolecki and Russel [6], Mizel and Seidman [9], [10], Seidmann [15] and other authors have represented certain results concerning observability for systems governed by linear parabolic partial differential equations with various boundary conditions. However, only observability for special cases of regular operators (see [2] for more general definitions) is considered in these investigations.

The Bessel differential operator has a discontinuity at zero and hence is of a singular type. Thus, in particular, results reported in [4], [15], cannot be applied directly to this problem. For regular operators, considered in references [4], [6] and [9], the modulus of the eigenfunctions do not tend to zero, and the distances between any two successive zeros of the eigenfunctions are equal. These two properties are not valid for the case involving Bessel's differential operator. Nevertheless, similar results can be established via a different approach. This is the main aim of this paper.

In Section 2, we describe the system and specify the problem to be considered. Some important preparatory results are also given in Section 3, a known sufficient continuity criterion for the operator $\phi_{\theta, T}$ is recalled briefly. This result is then used to prove another sufficient continuity criterion for $\phi_{\theta, T}$. Furthermore, an expression for the observation time is also stated. In Section 4, a Theorem on the a.e. existence of a continuous linear operator $\phi_{\theta, T}$ is proved for a special case of the considered system. Using this result we show that the set of $\theta \in[0,1]$ for which $\phi_{\theta, T}$ does not exist (for a fixed $T>0$ ) is dense in $[0,1]$. In the final Section 5 , we extend the main result to the case in which the system is described by the Bessel differential operator of order $\nu$ with $\nu \in\left[\frac{1}{2}, 1\right]$.

\section{Problem statement and preparatory results}

Let $T$ be a fixed positive real number and consider for each $\nu \geqslant \frac{1}{2}$ or $\nu=0$ the linear parabolic partial differential equation

$$
u_{t}+B_{(0,1)}^{\nu} u=0, \quad(r, t) \in(0,1) \times(0, T),
$$


where $B_{(0,1)}^{\nu}$ is the Bessel differential operator of order $\nu$, defined by

$$
B_{(0,1)}^{\nu} \psi \equiv-\frac{1}{r} \frac{d}{d r}\left(r \frac{d \psi}{d r}\right)+\frac{\nu^{2}}{r^{2}} \psi
$$

To this partial differential equation three types of boundary conditions, namely

$$
\begin{aligned}
u(1, t) & =0, \\
\frac{\partial u}{\partial r}(1, t) & =0, \\
\frac{\partial u}{\partial r}(1, t)+\sigma u(1, t) & =0, \quad \sigma>0 \text { fixed, }
\end{aligned}
$$

together with $u(0+)$ bounded in all three cases for each $\nu \geqslant \frac{1}{2}$ or $\nu=0$, are considered. The initial condition is

$$
u(r, 0)=u_{0}(r)
$$

where $u_{0}$ is an element in the Hilbert space $H$ defined by

$$
H \equiv L^{2}(0,1 ; \sqrt{r}) \equiv\left\{x: \sqrt{r} x(r) \in L^{2}(0,1)\right\} .
$$

In this paper, we consider three types of problems. Each of them is formulated from equation (2.1), where $\nu=0$ or $\nu \in\left[\frac{1}{2}, 1\right]$, by including the initial condition (2.5) together with one of the boundary conditions. For brevity, these three problems are to be called problem 1, problem 2 and problem 3 respectively.

Let $J_{\nu}$ be the Bessel function of order $\nu$ and let

$$
f_{\nu, j} \equiv d_{\nu, j} J_{\nu}\left(\sqrt{\lambda_{\nu, j}} r\right), \quad j=1,2, \ldots,
$$

where $\lambda_{\nu, j} \equiv \mu_{\nu, j}^{2}$ denote the eigenvalues for each of the problems 1,2 and 3. For each problem, let $\left\{f_{\nu, j}(r)\right\}$ be the sequence of the corresponding eigenfunctions which is orthonormal with respect to the natural norm in $H$. Let $C_{v, j}, j=1,2, \ldots$, be the Fourier-Bessel-Dini coefficients of $u_{0}$ with respect to the natural scalar product in $H$. It is well known that

$$
u_{\nu}(r, t)=\sum_{j=1}^{\infty} C_{\nu, j} e^{-\lambda_{\nu, j} t} f_{\nu, j}(r)
$$

belongs to $C^{2}((0,1) \times(0, T))$ and is the unique solution for each corresponding problem to the considered $\nu$.

REMARK 2.1. The explicit expressions for the three different eigenfunctions $f_{\nu, j}(r)$, can be found in [7, Section 7.10.4]. Furthermore, it is known [1, expression 9.1.60] that

$$
\left|J_{v}(r)\right| \leqslant 1, \quad \forall r \in \mathbf{R}, \nu \geqslant 0 .
$$

Without loss of generality, the expression for $f_{v, j}$ given in (2.6) is normalized with respect to the norm in $C[0,1]$. 
Before defining our observation problem, we need some notation.

Let $X, Y, Z$ be Banach spaces. Consider the abstract linear system

$$
\begin{aligned}
& X \supset \mathscr{D}(C) \stackrel{\stackrel{C}{\rightarrow}}{\rightarrow} Y \\
& F \searrow \\
& Z
\end{aligned}
$$

where the observation operator $C: X \rightarrow Y$ is linear with dense domain $\mathscr{D}(C)$ and $F$ is linear and bounded.

With this notation we give a general definition of observation problems.

Definition 2.2. The system (2.8) is (continuously) F-observable if there exists a constant $K \geqslant 0$ such that

$$
\|F x\|_{Z} \leqslant K\|C x\|_{Y}, \quad x \in \mathscr{D}(C) .
$$

The system (2.8) is F-constructible if there is a bounded linear operator $G: Y \rightarrow Z$ such that

$$
F=G C,
$$

i.e. $G$ makes the diagram (2.8) commutative.

In application, we may choose $X=H$, the Hilbert space of the initial conditions, $Y=C[\alpha, T]$ or $L^{p}[\alpha, T], p \in[2, \infty)$ with $0<\alpha<T$, and $Z=H$ or $C[0,1]$. Let $\theta \in[0,1]$ be arbitrary but fixed. Define the observation operator $C: X \rightarrow Y$ by

$$
C_{\theta} u_{0}=u(\theta, \cdot)
$$

Then, $C_{\theta}$ is a continuous linear operator, since by Remark 2.1 we have for $\nu \geqslant 0$ :

$$
\begin{aligned}
\left\|C_{\theta} u_{0}\right\|_{Y} & \leqslant \max (1, T)\|u(\theta, \cdot)\|_{C[\alpha, T]} \\
& \leqslant \max (1, T) \tilde{K}_{\nu}\left(\sum_{j=1}^{\infty}\left|C_{\nu, j}\right|^{2}\right)^{1 / 2}=\max (1, T) \tilde{K}_{\nu}\left\|u_{0}\right\|_{H},
\end{aligned}
$$

where

$$
\tilde{K}_{\nu} \equiv\left(\sum_{j=1}^{\infty} e^{-2 \lambda_{r, j} \alpha}\right)^{1 / 2}<\infty, \quad \alpha>0 .
$$

Clearly, $C_{\theta}$ is linear, while the finiteness of $\tilde{K}_{v}$ follows from Lemma 2.3.

The final state operator $F: X \rightarrow Z$ is defined by $F u_{0}=u(\cdot, T) . F$ is linear and with exactly the same arguments as above one shows that $F$ is continuous. The reconstruction operator $G=\phi_{\theta, T}: Y \rightarrow Z$ is therefore defined by $\phi_{\theta, T} u(\theta, \cdot)=$ $u(\cdot, T)$. 
From Definition 2.2, the reconstruction problem consists in determining a continuous linear operator $\phi_{\theta, T}$, depending on $\theta$ and $T$, which makes the diagram (2.8) commutative, e.g. $F u_{0}=\phi_{\theta, T} C_{\theta} u_{0}$.

Note that the continuity of the operator $\phi_{\theta, T}$ implies final state observability,

$$
\left\|F u_{0}\right\|_{Z}=\left\|\phi_{\theta, T} C_{\theta} u_{0}\right\|_{Z} \leqslant K\left\|C_{\theta} u_{0}\right\|_{Y} .
$$

For convenience, certain results concerning the roots of the eigenvalues for each of the problems $1,2,3$ are summarized in

LEMMA 2.3. For each $j=1,2, \ldots$, and fixed $\nu=0$ or $\nu \geqslant \frac{1}{2}$, let $\mu_{\nu, j}=\sqrt{\lambda_{\nu, j}}$ denote any of $p_{\nu, j}, y_{\nu, j}$ or $\gamma_{\nu, j}$, where $p_{\nu, j}$ (respectively $y_{\nu, j}$ and $\gamma_{\nu, j}$ ) is the root of the eigenvalue of the problem 1 (respectively problem 2 and problem 3). Then it is true for each $\nu=0$ or $\nu \geqslant \frac{1}{2}$, that

$$
\lim _{j \rightarrow \infty}\left(\mu_{p, j+1}-\mu_{\nu, j}\right)=\pi
$$

and

$$
\mu_{\nu, j}=O(j) .
$$

Let $\nu>\frac{1}{2}$. For all three cases of boundary conditions (2.2), (2.3), and (2.4) (except the case $\mathrm{\sigma}=\frac{1}{2}$ in (2.4)), the inequalities

$$
\mu_{\nu, 2}-\mu_{\nu, 1}>\cdots>\mu_{\nu, j+1}-\mu_{\nu, j}>\cdots>\rightarrow \pi
$$

are valid.

If $\nu=\frac{1}{2},(2.12)$ is also true in the case of the boundary condition (2.3). For the boundary conditions (2.2) and (2.4) with $\sigma=\frac{1}{2}$ all inequalities in (2.12) become equalities.

For $\nu=0$, it is true that

$$
\begin{aligned}
& \left(j-\frac{1}{4}\right) \pi \leqslant p_{0, j} \leqslant\left(j-\frac{1}{8}\right) \pi, p_{j+1}-p_{j}<\pi, \quad \forall j \in \mathbf{N}, \\
& \left(j-\frac{7}{8}\right) \pi \leqslant y_{0, j} \leqslant\left(j-\frac{3}{4}\right) \pi, y_{0, j+1}-y_{0, j}>\pi, \quad y_{0,1}=0 \text {, } \\
& \gamma_{0, j}=j \pi+q+\psi(j) / j, \quad \forall j \in \mathbf{N},
\end{aligned}
$$

where $q$ is a constant and $\psi(j)$ is a suitable bounded function in $\mathbf{N}$.

PRoof. a) $\mu_{\nu, j}=p_{\nu, j}$ : In the case $\nu \geqslant \frac{1}{2},(2.10)$ and (2.12) are proved in [8, Theorem 1.4 and 1.2].

For $\nu=0,(2.10)$ and (2.13) are known (see [14, page 43]).

b) $\mu_{\nu, j}=y_{\nu, j}$ : Let $\nu \geqslant \frac{1}{2}$. The proof of (2.12) is then a special case $(\sigma=\alpha=0)$ in the proof of Theorem 1.1 in [8]. For (2.10) see Theorem 1.4 in that reference. 
In the case $\nu=0$ we get $y_{0, j}=p_{1, j}$ since $J_{0}^{\prime}(r)=-J_{1}(r)$. The validity of (2.10) and (2.12) follows then from (a). For a proof of the first inequality in (2.14) see [13, page 314].

c) $\mu_{\nu, j}=\gamma_{\nu, j}$ : For a proof of (2.10) and (2.12) in the case of $\nu \geqslant \frac{1}{2}, \sigma>0$, see [8, Theorem 1.4 and 1.1]. Equation (2.15) follows from equation (57) of [11, page 406]. The expression (2.10), in this case $\nu=0$, follows then directly from (2.15).

Because all intervals between two successive positive roots $\mu_{\nu, j}$ equal or exceed $\pi$, it follows that

$$
\mu_{\nu, j} \geqslant(j-1) \pi, \quad j=1,2, \ldots,
$$

except in the two cases of (2.13) and (2.15), where in case of (2.15) such a result is not stated. But, $\mu_{\nu, j} \geqslant(j-1) \pi$ together with the first inequality in (2.13) respectively (2.15) in the exceptional cases imply that $\mu_{\nu, j}=O(j)$ for each $\nu \geqslant \frac{1}{2}$ or $\nu=0$. This completes the proof.

The next lemma is needed in Section 3 for the establishment of a sufficient criterion for the continuity of the operator $\phi_{\theta, T}$.

Lemma 2.4. Let $\nu \geqslant 0$. For sufficiently large $\lambda \in \mathbf{R}^{+}$, the following inequality is valid:

$$
\frac{K(\nu)}{\lambda} \leqslant\left\|J_{\nu}(\lambda x)\right\|_{H}^{2}<\frac{M(\nu)}{\lambda},
$$

where $M(\nu)$ and $K(\nu)>0$ are constants which depend on $\nu$.

Proof. See the paragraph 14 in Section 13 of [16].

\section{Continuity criteria}

Let $\left\{\tilde{\lambda}_{j}\right\}, j=1,2, \ldots$, be a sequence of complex numbers. Furthermore, it is assumed that the following conditions are satisfied:

$$
\begin{gathered}
\sum_{j=1}^{\infty}\left|\tilde{\lambda}_{j}\right|^{-1}<\infty, \\
\exists p>0, \forall i, j=1,2, \ldots,\left|\tilde{\lambda}_{j}-\tilde{\lambda}_{i}\right| \geqslant p|i-j|, \\
\exists \delta>0, \exists j_{0}, \forall j \geqslant j_{0}, \operatorname{Re} \tilde{\lambda}_{j} \geqslant \delta\left|\tilde{\lambda}_{j}\right| .
\end{gathered}
$$

THEOREM 3.1. Let $\left\{\tilde{\lambda}_{j}\right\}, j=1,2, \ldots$, be a sequence of complex numbers satisfying (3.1)-(3.3). Under the following conditions: there exists a sequence $\left\{B_{j}\right\}, j=1,2, \ldots$, 
of real numbers such that

a)

$$
\left\|f_{j}\right\|_{Z} \leqslant B_{j}\left|f_{j}(\theta)\right|, \quad \theta \in[0,1]
$$

and there is an $\varepsilon>0$ so that

b)

$$
\sum_{j=1}^{\infty} B_{j} \exp \left(-(T-\varepsilon) \tilde{\lambda}_{j}\right)<\infty
$$

$\phi_{\theta, r}: Y \rightarrow Z$ is well-defined and bounded.

Proof. [4, Lemma 1] or [12, Satz 7.2].

From Lemma 2.3, it follows immediately that one can set $\left\{\tilde{\lambda}_{j}\right\}=\left\{\lambda_{\nu, j}\right\}$, $j=1,2, \ldots$, in the expressions (3.1)-(3.3) for each fixed number $\nu \geqslant \frac{1}{2}$ or $\nu=0$. Consequently, the operator $\phi_{\theta, T}$ makes the diagram (2.8) commutative if we can verify the conditions (a) and (b) of Theorem 3.1.

For brevity, let $\langle Y, Z\rangle$ denote any pair of Banach spaces $Y=C[\alpha, T]$ or $L^{p}[\alpha, T], p \in[2, \infty), 0<\alpha<T$, and $Z=H$ or $C[0,1]$.

THEOREM 3.2. a) If $\sum_{j=1}^{\infty} \exp \left(-\lambda_{\nu, j} T\right)\left|f_{\nu, j}(\theta)\right|^{-1}$ is convergent, $\nu \geqslant \frac{1}{2}$ or $\nu=0$, then $\phi_{\theta, T^{\prime}}$ is well-defined and bounded for $T^{\prime}>T>0$ and for any pair $\langle Y, Z\rangle$.

b) If this series is divergent, then $\phi_{\theta, T^{\prime}}$ is unbounded for $T^{\prime}<T$ and for any pair $\langle Y, Z\rangle$.

Proof. a) Set $B_{j} \equiv\left|f_{\nu, j}(\theta)\right|^{-1}$ for an arbitrary but fixed $\nu \geqslant \frac{1}{2}$ or $\nu=0$ and apply Theorem 3.1 .

b) Clearly, if this series is divergent ( $\nu$ fixed), then there does not exist a $j_{0} \in \mathbf{N}$ such that for all $j \geqslant j_{0}$,

$$
\exp \left(-\lambda_{\nu, j} T\right)\left|f_{\nu, j}(\theta)\right|^{-1}<(j)^{-3 / 2} .
$$

Thus, we can find a subsequence $\left\{j_{n}\right\}$ of the sequence $\{j\}$ so that, after multiplying with $j_{n}^{3}$,

$$
\exp \left(-\lambda_{\nu, j_{n}}(T-\varepsilon)\right)\left|f_{v, j}(\theta)\right|^{-1} \geqslant\left(j_{n}\right)^{3 / 2}
$$

for sufficiently large $j_{n}$. To show that $\phi_{\theta, T}$ is unbounded, it suffices to show that there is a subsequence, again indexed by $j_{n}$, such that

$$
\psi\left(j_{n}\right)=\frac{\left\|\phi_{\theta, T}\left(\exp \left(-\lambda_{\nu, j_{n}} t\right)\right)\right\|_{Z}}{\left\|\exp \left(-\lambda_{\nu, j_{n}} t\right)\right\|_{Y}} \rightarrow \infty, \quad \text { as } j_{n} \rightarrow \infty .
$$


For this, we note that, if $Y=L^{p}[\alpha, T], p \in[2, \infty)$, then

$$
\left\|\exp \left(-\lambda_{\nu, j} t\right)\right\|_{Y} \leqslant 1 \text { as } j \rightarrow \infty \text {. }
$$

On the other hand, the inequality (3.4) becomes an equality if $Y=C[\alpha, T]$ and $\alpha \rightarrow 0$. Thus, by definition of $\phi_{\theta, T}$,

$$
\psi(j) \geqslant \frac{\exp \left(-\lambda_{\nu, j} T^{\prime}\right)}{\left|f_{\nu, j}(\theta)\right|}\left\|f_{\nu, j}\right\|_{Z}
$$

Since $\left\|f_{\nu, j}\right\|_{C[0,1]} \geqslant\left\|f_{\nu, j}\right\|_{H}$, it follows from Lemma 2.4 and (3.5) that

$$
\psi(j) \geqslant \frac{\exp \left(-\lambda_{\nu, j} T^{\prime}\right)}{\left|f_{\nu, j}(\theta)\right|}\left(\frac{K(\nu)}{\sqrt{\lambda_{\nu, j}}}\right)^{1 / 2} \geqslant j^{3 / 2}\left(\frac{K(\nu)}{\sqrt{\lambda_{\nu, j}}}\right)^{1 / 2} \geqslant c(\nu) j \rightarrow \infty,
$$

as $j \rightarrow \infty$, where $c(\nu)$ is a constant which depends on the chosen $\nu, \lambda_{\nu, j}=O\left(j^{2}\right)$ and $T^{\prime}$ is chosen as $T-\varepsilon$.

As a consequence of Theorem 3.2, we have

Corollary 3.3. For any $\theta \in[0,1]$, if $f_{\nu, j}(\theta) \neq 0$ for all $j \in \mathbf{N}$, where $\nu \geqslant \frac{1}{2}$ or $\nu=0$, the observation time $T_{0}$ is given by

$$
T_{0}(\theta)=-\lim _{j \rightarrow \infty} \frac{\log \left|f_{\nu, j}(\theta)\right|}{\lambda_{\nu, j}} .
$$

If there exists a $j \in \mathbf{N}$ such that $f_{\nu, j}(\theta)=0$ then $\phi_{\theta, T}$ is not well-defined. In this case, set $T_{0}=\infty$.

Proof. See Corollary 1 of [4].

\section{Main theorem}

We state our main result for the case $\nu=0$ in

TheORem 4.1. For almost all $\theta \in[0,1]$ and for any pair $\langle Y, Z\rangle$ there exists a continuous linear operator $\phi_{\theta, T}(T>0)$ which makes the diagram (2.8) commutative.

For the proof of this theorem, we need some preparations.

Let $P \equiv\left\{p_{j}, j \in \mathbf{N}_{0}\right\}$ be the set of the positive zeros of $J_{0} \cup\left\{p_{0}=0\right\}$ and define for $r \geqslant 0$,

$$
\delta(r, P) \equiv \inf _{j \in \mathrm{N}_{0}}\left\{\left|r-p_{j}\right|: p_{j} \in P\right\} .
$$

Next, we need a lemma which is related to the diophantine approximation theory. 
LEMMA 4.2. Let $b, w$ be real numbers with $w>\pi$ and $0 \leqslant b \leqslant \frac{1}{4}$. Let $\delta$ be defined as in (4.1). Then, the Lebesgue measure $\lambda$ of the set

$$
H_{w, b} \equiv\{\theta \in[0,1]: \delta(w \theta, P) \leqslant b\}
$$

is less than or equal to $4 b$, that is $\lambda\left(H_{w, b}\right) \leqslant 4 b$.

Proof. From the definition of $H_{w, b}$, it is clear that

$$
H_{w, b} \subset \bigcup_{j=1}^{\infty}\left[\frac{p_{j}-b}{w}, \frac{p_{j}+b}{w}\right] .
$$

Note that each of the subintervals in (4.2) has length $2 b / w$, and that each distance between any two successive subintervals has length $\left(p_{j+1}-p_{j}-2 b\right) / w$. Suppose that $K$ subintervals in (4.2) have nonempty intersection with [0,1]. Since $w>\pi>p_{1} \approx 2.4 \ldots$ (see [1, Table 9.5]) and $0 \leqslant b \leqslant \frac{1}{4}$, it follows that $K \geqslant 2$. Thus,

$$
\lambda\left(H_{w, b}\right) \leqslant K\left(\frac{2 b}{w}\right) .
$$

Note that $p_{1}-p_{0}=p_{1}$ and $p_{1}-2 b>1$. Furthermore, from (2.13), $p_{j+1}-p_{j}$ $\geqslant \frac{7}{8} \pi$ for all $j \in \mathbf{N}$. Thus, together with (4.3), we have

$$
\begin{aligned}
\lambda\left([0,1] \backslash H_{w, b}\right) & \geqslant(K-1)\left(\frac{p_{j+1}-p_{j}-2 b}{w}\right) \\
& \geqslant(K-1)\left(\frac{p_{1}-2 b}{w}\right) \geqslant \frac{K-1}{w} .
\end{aligned}
$$

Combining (4.3) and (4.4), we get

$$
\frac{\lambda\left(H_{w, b}\right)}{\lambda\left([0,1] \backslash H_{w, b}\right)} \leqslant \frac{2 K b}{K-1} \leqslant 4 b .
$$

This completes the proof.

The next lemma follows from Lemma 4.2 and the Borel-Cantelli Lemma.

LEMMA 4.3. Let $\left\{b_{j}\right\},\left\{\mu_{j}\right\}, j=1,2, \ldots$, be sequences of real numbers such that $b_{j}>0, \forall j \in \mathbf{N}, \underline{\lim }_{j \rightarrow \infty} \mu_{j}>\pi$, and $\sum_{j=1}^{\infty} b_{j}<\infty$. Then,

$$
\lambda(H) \equiv \lambda\left\{\theta \in[0,1]: \forall N, \exists j>N: \delta\left(\mu_{j} \theta, P\right) \leqslant b_{j}\right\}=0 .
$$

To proceed further, it is useful to introduce the function

$$
w_{0}(r) \equiv \sqrt{\frac{\pi r}{2}}\left|J_{0}(r)\right|, \quad r \geqslant 0 .
$$


LEMMA 4.4. Let $w_{0}(r)$ and $\delta(r, P)$ be defined by (4.5) and (4.1) respectively. Then, there exists a constant $c, 0<c<1$, such that for all $r \geqslant 0$,

$$
w_{0}(r) \geqslant c \delta(r, P) \text {. }
$$

Proof. Since $J_{0}(r)$ satisfies the Bessel differential equation of zeroth order it is easily checked that $w_{0}(r)$ is strictly concave in each of the intervals $\left(p_{j}, p_{j+1}\right)$, $j \in \mathbf{N}_{0}$. Now, let $q_{j}, j \in \mathbf{N}_{0}$, denote the point in $\left(p_{j}, p_{j+1}\right)$ where $w_{0}(r)$ attains its maximum in $q_{j}$ over the interval $\left(p_{j}, p_{j+1}\right)$. Then,

$$
w_{0} \geqslant \frac{\left|J_{0}\left(q_{j}\right) \sqrt{q_{j} \pi / 2}\right|}{q_{j}-p_{j}}\left(r-p_{j}\right), \quad r \in\left[p_{j}, q_{j}\right],
$$

and

$$
w_{0}(r) \geqslant \frac{\left|J_{0}\left(q_{j}\right) \sqrt{q_{j} \pi / 2}\right|}{p_{j+1}-p_{j}}\left(p_{j+1}-r\right), \quad r \in\left[q_{j}, p_{j+1}\right] .
$$

These in turn imply that

$$
w_{0}(r) \geqslant \sqrt{\frac{\pi}{2}} \frac{\inf _{j \in \mathbf{N}_{0}}\left|J_{0}\left(q_{j}\right) \sqrt{q_{j}}\right|}{\sup _{j \in \mathbf{N}_{0}}\left(p_{j+1}-p_{j}\right)} \delta(r, P) .
$$

From equality (2.13) and the facts that $q_{0} \leqslant p_{1} \approx 2.4 \ldots,\left|J_{0}(x)\right| \leqslant 1, x \in \mathbf{R}$, it follows that the constant $c$ can be chosen:

$$
0<c \equiv \sqrt{\frac{\pi}{2}} \frac{1}{\pi} \inf _{j \in \mathbf{N}_{0}}\left|J_{0}\left(q_{j}\right) \sqrt{q_{j}}\right| \leqslant\left(\frac{1}{2 \pi}\right)^{1 / 2} \sqrt{q_{0}}<1 .
$$

LEMMA 4.5. Let $\left\{\mu_{j}\right\}, j=1,2, \ldots$, be a sequence of positive real numbers with $\lim _{j} \inf \left(\mu_{j} / j\right)>0$. Then, there exists a set $\Theta \subset[0,1]$ with $\lambda(\Theta)=1$ such that, for all $\theta \in \Theta$,

$$
J_{0}\left(\mu_{j} \theta\right) \neq 0, \quad \forall j \in \mathbf{N}
$$

and

$$
\left\|J_{0}\left(\mu_{j} r\right)\right\|_{Z} \leqslant B_{j, \theta}\left|J_{0}\left(\mu_{j} \theta\right)\right|, \quad \forall j \in \mathbf{N}
$$

where

$$
B_{j, \theta} \equiv\left|J_{0}\left(\mu_{j} \theta\right)\right|^{-1}
$$

and

$$
\sum_{j=1}^{\infty} B_{j, \theta} \exp \left(-\mu_{j}^{2} T\right)<\infty \text { for } T>0
$$


Proof. Let

$$
\begin{aligned}
\Theta \equiv & \left\{\theta \in[0,1]: \exists N=N(\theta), \forall j \geqslant N: \delta\left(\mu_{j} \theta, P\right) \geqslant \mu_{j}^{5 / 2} \exp \left(\mu_{j}^{2} T\right)\right\} \\
& \cap\left\{\theta \in[0,1]: \exists j \in \mathbf{N}_{0} \text { with } \mu_{j} \theta \in P\right\}^{c} \equiv A \cap B^{c},
\end{aligned}
$$

where $B^{c}$ denotes the complement of the set $B$.

Since $B$ represents the countable set of the zeros of $w_{0}(r)$, the validity of $(4.7)$ is clear. On this basis, it follows from Lemma 4.3 that $\lambda(\theta)=1$.

For $\theta \in \Theta, B_{j, \theta}$ is well-defined which together with (2.7) implies (4.8).

Let $\theta \in \Theta$, and $T>0$. Then,

$$
\begin{aligned}
& \sum_{j=1}^{\infty} B_{j, \theta} \exp \left(-\mu_{j}^{2} T\right) \\
& \leqslant \sum_{j=1}^{N(\theta)} \exp \left(-\mu_{j}^{2} T\right)\left|J_{0}\left(\mu_{j} \theta\right)\right|^{-1}+\sum_{j=N(\theta)+1}^{\infty} \exp \left(-\mu_{j}^{2} T\right)\left|J_{0}\left(\mu_{j} \theta\right)\right|^{-1} \\
& \quad \leqslant C(\theta)+\frac{1}{c} \sqrt{\frac{\pi}{2}} \sum_{j=N(\theta)+1}^{\infty} \frac{\sqrt{\mu_{j}^{\theta}} \exp \left(-\mu_{j}^{2} T\right)}{\delta\left(\mu_{j} \theta, P\right)} \quad \text { (using inequality (4.6)) } \\
& \leqslant C(\theta)+\frac{1}{c} \sqrt{\frac{\pi}{2} \theta} \sum_{j=N(\theta)+1}^{\infty} \mu_{j}^{-2}<\infty \quad \text { (from the definition of } \Theta \text { ), }
\end{aligned}
$$

where $N(\theta)$ is an integer and $C(\theta)$ is a constant both depending on $\theta$.

The proof is complete.

We are now in a position to prove Theorem 4.1.

Proof of Theorem 4.1. The eigenfunctions of each of the problems 1,2 and 3 are represented by $f_{j}(r) \equiv J_{0}\left(\mu_{0, j} r\right)$ and the corresponding eigenvalues are $\lambda_{j} \equiv$ $\mu_{0, j}^{2}, j=1,2, \ldots$ Furthermore, it is obvious that $\phi_{\theta, T}$ is a linear operator. Thus, the result follows from Theorem 3.1 and Lemma 4.5.

This completes the proof.

As a consequence of Theorem 4.1, we have

COROllary 4.6. Let $T_{0}(\theta)$ be as defined in (3.6). Then,

$$
\lambda\left\{\theta \in[0,1]: T_{0}(\theta)=0\right\}=1 .
$$

REMARK 4.7. Using a similar technique given for Theorem 4 of [4], it can be shown that the result of Corollary 4.6 implies that of Theorem 4.1. Thus, these two results are equivalent. 
REMARK 4.8. In the case $J_{0}\left(\mu_{j} \theta\right)=0$ for $a j \in \mathbf{N}$ and $\theta \in[0,1]$, it is clear (see Theorem 3.2b) that there exist no continuous linear operators $\phi_{\theta, T}$ which make the diagram (2.8) commutative. In the next Theorem we shall prove that the set

$$
H \equiv\left\{\theta \in[0,1]: \exists j \in \mathbf{N} \text { with } J_{0}\left(\mu_{j} \theta\right)=0\right\}
$$

is dense in $[0,1]$. Therefore, the set of all $\theta \in[0,1]$ for which $\phi_{\theta, T}$ is not bounded for $T>0$ and for any pair $\langle Y, Z\rangle$ is dense in $[0,1]$ and has nevertheless Lebesgue measure zero according to Theorem 4.1.

THEOREM 4.9. Let for each $\nu \geqslant \frac{1}{2}$ or $\nu=0,\left\{\mu_{\nu, j}\right\}, j=1,2, \ldots$, denote the sequence of the positive zeros of $J_{\nu}(r)$. Then, the set

$$
\left\{\frac{\mu_{\nu, j}}{\mu_{\nu, n}}: j, n \in \mathbf{N}, n \geqslant j\right\}
$$

is dense in $[0,1]$.

Proof. It suffices to show that $\forall p, q \in \mathbf{N}(p \leqslant q), \exists j, n \in \mathbf{N}(n \geqslant j)$ such that

$$
\frac{p}{q} \leqslant \frac{\mu_{v, j}}{\mu_{\nu, n}} \leqslant \frac{p+1}{q},
$$

for each arbitrary but fixed $\nu \geqslant \frac{1}{2}$ or $\nu=0$.

Choose an $n \in \mathbf{N}$ so that for arbitrary but fixed $p, q(p \leqslant q)$,

$$
\frac{\mu_{\nu, n}}{\mu_{\nu, 1}} \leqslant 2 q \leqslant \frac{\mu_{\nu, n+1}}{\mu_{\nu, 1}},
$$

respectively

$$
\frac{\mu_{0, n}}{\pi} \leqslant 2 q \leqslant \frac{\mu_{0, n+1}}{\pi}, \quad(\nu=0) .
$$

To this $n \in \mathbf{N}$, we choose a $j>1$ such that

$$
\begin{gathered}
\mu_{\nu, j-1} \leqslant \mu_{\nu, n} p / q \leqslant \mu_{\nu, j}, \\
0<\mu_{\nu, n} p / q<\mu_{\nu, 1} \text { for } j=1,
\end{gathered}
$$

where the existence of such integers $n, j \geqslant 1$ follow from the inequalities $0<\mu_{\nu, 1}$ $<\mu_{\nu, 2}<\cdots<\mu_{\nu, j} \rightarrow \infty$ for $j \rightarrow \infty$ and $\pi \leqslant \mu_{\nu, j+1}-\mu_{\nu, j} \leqslant \mu_{\nu, 1}, j \in \mathbf{N}$, except in the case of $\nu=0$, where $\frac{7}{8} \pi \leqslant \mu_{0, j+1}-\mu_{0, j}<\pi$ (see (2.13)). Inequalities (4.13a) and (2.12) (respectively (2.13)) together with $\mu_{\nu, n} \geqslant q \mu_{\nu, 1}$ (see 4.12a) (respectively $\mu_{0, n} \geqslant q \pi$ ) imply that

$$
q \mu_{\nu, j} \leqslant \mu_{\nu, n}(p+1) .
$$

Combining (4.13a) and (4.14), the inequality (4.11) is proved for $\nu \geqslant \frac{1}{2}, \nu=0$ and $j>1$. For (4.13b) observe that $q \mu_{v, 1} \leqslant p \mu_{\nu, n}+q \mu_{v, 1} \leqslant \mu_{v, n}(p+1)$, where we used the fact $\mu_{0,1}<\pi$ in the case of $\nu=0$.

Thus, the proof is complete. 


\section{Certain extensions}

In this section, the main result reported in Theorem 4.1 is extended to the case in which the system (2.1) is described by the Bessel differential operator of order $\nu$ with $\nu \in\left[\frac{1}{2}, 1\right]$ rather than $\nu=0$. We shall only indicate the major changes in the proof.

Let $P_{\nu} \equiv\left\{p_{\nu, j}, j \in \mathbf{N}_{0}\right\}$ be the set of the zeros of $J_{\nu}$, where $\nu \in\left[\frac{1}{2}, 1\right]$, and define, for $r \geqslant 0$,

$$
\delta\left(r, P_{\nu}\right) \equiv \inf _{j \in \mathrm{N}_{0}}\left\{\left|r-p_{\nu, j}\right|: p_{\nu, j} \in P_{\nu}\right\}
$$

LeMma 5.1. For $\nu \in\left(\frac{1}{2}, 1\right]$, define

$$
w_{\nu}(r) \equiv\left\{\begin{array}{l}
\sqrt{\pi r / 2}\left|J_{\nu}(r)\right|, \quad \text { for } r \in\left[p_{\nu, j}, p_{\nu, j+1}\right], j=1,2, \ldots, \\
J_{\nu}(r), \quad \text { for } r \in\left[0, p_{\nu, 1}\right] .
\end{array}\right.
$$

For $\nu=\frac{1}{2}$, define

$$
w_{1 / 2}(r) \equiv \sqrt{\pi r / 2}\left|J_{1 / 2}(r)\right|, \quad r \geqslant 0 .
$$

Then, $w_{\nu}(r), \nu \in\left[\frac{1}{2}, 1\right]$, is strictly concave in each of the intervals $\left[p_{\nu, j}, p_{\nu, j+1}\right]$, $j=1,2, \ldots ;$ and $w_{1 / 2}(r)$ is strictly concave in $\left(0, p_{1 / 2,1}\right)$ too.

Proof. For each $\nu \in\left(\frac{1}{2}, 1\right]$, consider intervals $\left(p_{\nu, j}, p_{\nu, j+1}\right], j \in \mathbf{N}$, where $J_{\nu}$ is positive. Using the recurrence relation (see [7], 7.2.8, expression (55))

$$
r J_{\nu}^{\prime}(r) \equiv \nu J_{\nu}(r)-r J_{v+1}(r),
$$

together with the Bessel differential equation, we obtain

$$
w_{\nu}^{\prime \prime}(r)=-r^{-3 / 2}\left(r^{2}-\nu^{2}+\frac{1}{4}\right) J_{\nu}(r) .
$$

Then, $w_{\nu}(r)$ is strictly concave in the considered intervals iff $r^{2}-\nu^{2}+\frac{1}{4}>0$ which is equivalent to $r>\sqrt{\nu^{2}-\frac{1}{4}}$, since $r \geqslant 0$. From [17, page 485], we observe that $p_{\nu, 1}>\sqrt{\nu(\nu+2)}>\sqrt{\nu^{2}-\frac{1}{4}}$. Thus, the desired result follows. The case when $J_{\nu}<0$ can be treated similarly.

Since $J_{1 / 2}(r)=\sqrt{2 / \pi r} \sin r$, (see [7], page 79, expression (1.4)), the concavity of $w_{1 / 2}(r)$ in the intervals $\left(p_{1 / 2, j}, p_{1 / 2, j+1}\right)=(j \pi,(j+1) \pi), j \in \mathbf{N}_{0}$ is clear.

The proof is complete.

REMARK 5.2. From the series representation of $J_{\nu}(r)$ for $\nu \geqslant 0$ (see [7], page 4, expression (2)), follows that $\lim _{r \rightarrow 0} J_{\nu}^{\prime}(r)=\infty$ for $\nu \in\left(\frac{1}{2}, 1\right)$ and $\lim _{r \rightarrow 0} J_{1}^{\prime}(r)=\frac{1}{2}$. Furthermore, we have $J_{v}^{\prime}\left(p_{v, 1}\right) \neq 0$, since there is only one positive zero of $J_{v}$. In fact, $J_{\nu}\left(p_{\nu, 1}\right)=J_{\nu}^{\prime}\left(p_{\nu, 1}\right)=0$ would imply that $J_{\nu}$ vanishes identically. However, this is 
impossible, since $J_{\nu}$ is a nontrivial solution of an ordinary differential equation of second order.

Using this result, (5.3) and the fact that $J_{\nu+1}(r)>0$ in $\left(0, p_{\nu, 1}\right)$ we conclude that $J_{\nu}^{\prime}\left(p_{\nu, 1}\right)<0$.

LEMMA 5.3. Let $w_{\nu}(r)$ and $\delta\left(r, P_{\nu}\right)$ be defined by (5.2a, b) and (5.11) respectively. Then, there exists a constant $c_{\nu}, 0<c_{\nu}<1$, such that, for all $r \geqslant 0$,

$$
w_{\nu}(r) \geqslant c_{\nu} \delta\left(r, P_{\nu}\right) \text {. }
$$

Proof. With the same proof as in Lemma 4.4, we get

$$
\begin{aligned}
w_{\nu}(r) & \geqslant \sqrt{\frac{\pi}{2}} \frac{\sqrt{q_{\nu, 1}}}{p_{\nu, 1}} \inf _{j \in \mathrm{N}}\left|J_{\nu}\left(q_{\nu, j}\right)\right| \delta\left(r, P_{\nu} \backslash\{0\}\right) \\
& \equiv c_{\nu}^{\prime} \delta\left(r, P_{\nu} \backslash\{0\}\right), \quad r \geqslant p_{\nu, 1},
\end{aligned}
$$

for each $\nu \in\left(\frac{1}{2}, 1\right)$, where

$$
0<c_{\nu}^{\prime} \leqslant \sqrt{\frac{\pi}{2}} \frac{\sqrt{2}}{\sqrt{p_{\nu, 1}}}<1 \quad \text { (using (2.2)). }
$$

In the case when $r \in\left[0, p_{\nu, 1}\right]$ with $\nu \in\left(\frac{1}{2}, 1\right]$, we proceed as follows: From Remark 5.2, we have $J_{\nu}^{\prime}(0)=\infty, J_{1}^{\prime}(0)=\frac{1}{2}$ and $J_{\nu}^{\prime}\left(p_{\nu, 1}\right)<0$. Now, by using the fact that the graph of $J_{\nu}(r)$ resembles a damped oscillation (see [3], pages $178-185)$, there exists a point $q_{\nu, 0} \in\left(0, p_{\nu, 1}\right)$ and a constant $a_{\nu}=a_{\nu}\left(q_{\nu, 0}\right)>0$ such that

$$
w_{\nu}(r) \geqslant \frac{a_{\nu}\left(q_{\nu, 0}\right)}{p_{\nu, 1}} \delta\left(r,\left\{0, p_{\nu, 1}\right\}\right) \equiv \tilde{c}_{\nu} \delta\left(r,\left\{0, p_{\nu, 1}\right\}\right), \quad r \in\left[0, p_{\nu, 1}\right]
$$

where $0<\tilde{c}_{\nu} \leqslant 1 / p_{\nu, 1} \leqslant 1 / \pi$.

Setting $c_{\nu} \equiv \min \left(c_{\nu}^{\prime}, \tilde{c}_{\nu}\right)$ and then combining (5.5) and (5.6) we get

$$
w_{\nu}(r) \geqslant c_{\nu} \delta\left(r, P_{v}\right) \text {. }
$$

The case when $\nu=\frac{1}{2}$ is trivial, since $P_{1 / 2}=\left\{n \pi, n \in \mathbf{N}_{0}\right\}$ and therefore,

$$
w_{1 / 2}(r) \geqslant \frac{2}{\pi} \delta\left(r, P_{1 / 2}\right) \text {. }
$$

The proof is complete.

With the same proof as for Lemma 4.2, by using the expression (2.12), we can show that the following remark is valid. 
REMARK 5.4. Let $\nu \in\left[\frac{1}{2}, 1\right]$ and let $b, \omega_{\nu}$ be real numbers with $\omega_{\nu}>p_{\nu, 1}$ and $0 \leqslant b \leqslant \frac{1}{4}$. Then,

$$
\lambda\left(H_{\omega_{v}, b}\right) \equiv \lambda\left\{\theta \in[0,1]: \delta\left(\omega_{\nu} \theta, P\right) \leqslant b\right\} \leqslant 4 b .
$$

For each $\nu \in\left[\frac{1}{2}, 1\right]$, define

$$
\begin{aligned}
\Theta_{\nu} \equiv & \left\{\theta \in[0,1]: \exists N=N(\theta), \forall j \geqslant N: \delta\left(\mu_{\nu, j} \theta, P_{\nu}\right) \geqslant \mu_{\nu, j}^{5 / 2} \exp \left(-\mu_{\nu, j}^{2} T\right)\right\} \\
& \cap\left\{\theta \in[0,1]: \exists j \in \mathrm{N}_{0} \text { with } \mu_{\nu, j} \theta \in P_{\nu}\right\}^{c} .
\end{aligned}
$$

Then, the proof of the expressions (4.7)-(4.9) for these values of $\nu$ is similar to that given for Lemma 4.5, except that (4.9) is required to be modified as follows:

Since only a finite number, say $M(\theta)$, of $\mu_{\nu, j} \theta \in\left(0, p_{\nu, 1}\right)$, we get

$$
\begin{aligned}
\sum_{j=1}^{\infty} B_{j, \theta} \exp \left(-\mu_{\nu, j}^{2} T\right) \leqslant & \sum_{j=1}^{N(\theta)} \exp \left(-\mu_{\nu, j}^{2} T\right)\left|J_{\nu}\left(\mu_{\nu, j} \theta\right)\right|^{-1} \\
& +\sum_{j=N(\theta)+1}^{M(\theta)} \exp \left(-\mu_{\nu, j}^{2} T\right)\left|J_{\nu}\left(\mu_{\nu, j} \theta\right)\right|^{-1} \\
& +\sum_{j=M(\theta)+1}^{\infty} \exp \left(-\mu_{\nu, j}^{2} T\right)\left|J_{\nu}\left(\mu_{\nu, j} \theta\right)\right|^{-1} \\
\leqslant & C(\theta)+\frac{1}{c_{\nu}} \sum_{j=N(\theta)+1}^{M(\theta)} \frac{\exp \left(-\mu_{\nu, j}^{2} T\right)}{\delta\left(\mu_{\nu, j} \theta,\left\{0, p_{\nu, 1}\right\}\right)} \\
& +\frac{1}{c_{\nu}} \sqrt{\frac{\pi \theta}{2}} \sum_{j=M(\theta)+1}^{\infty} \frac{1}{\mu_{\nu, j}^{2}}<\infty,
\end{aligned}
$$

for $\theta \in \Theta_{\nu}$; where $M(\theta), N(\theta)$ are integers and $C(\theta)$ is a constant, all depending on $\theta$ and $\nu$.

Now, the proof of the main result follows easily from the last result, the corresponding expressions (4.7), (4.8), and Theorem 3.1.

REMARK 5.5. The technique of this paper works only for the cases $\nu=0$ or $\nu \in\left[\frac{1}{2}, 1\right]$. The reason is that the technique requires that $J_{\nu}^{\prime}(0) \neq 0$, which is not true for $\nu>1$.

\section{Acknowledgment}

The author wishes to express his most sincere appreciation to Dr. K. L. Teo for reading the entire manuscript and giving him most valuable comments and suggestions. 


\section{References}

[1] M. Abramowitz and I. A. Stegun, Handbook of mathematical functions (Dover, New York, 1968).

[2] N. V. Ahmed and K. L. Teo, Optimal control of distributed parameter systems (North Holland, New York, 1981).

[3] R. G. Cooke, "Gibb's phenomenon in Fourier-Bessel series and integrals", Proc. London Math. Soc. 27 (1927), 171-192.

[4] S. Dolecki, "Observability for the one-dimensional heat equation", Studia Math. 48 (1973), 291-305.

[5] S. Dolecki, "Observability for regular processes", J. Math. Anal. Appl. 58 (1977), 178-188.

[6] S. Dolecki and D. L. Russel, "A general theory of observation and control", SIAM J. Control Optim. 15 (1977), 185-220.

[7] A. Erdèlyi, W. Magnus, F. Oberhettinger and F. G. Tricomi, Higher transcendental functions, Vol. II (McGraw-Hill, New York, 1953).

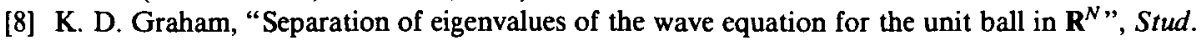
Appl. Math. 52 (1973), 329-343.

[9] V. J. Mizel and T. I. Seidman, "Observation and prediction for the heat equation", J. Math. Anal. Appl. 28 (1969), 303-312.

[10] V. J. Mizel and T. I. Seidmann, "Observation and prediction for the heat equation II", J. Math. Anal. Appl. 38 (1972), 149-166.

[11] C. N. Moore, "Summability of developments in Bessel functions", Trans. Amer. Math. Soc. 10 (1909), 391-435.

[12] S. Rolewicz, Funktionalanalysis und Steuerungstheorie (Springer, Berlin, 1976).

[13] P. Schafheitlin, "Die Nullstellen der Besselschen Funktionen", J. Reine Angew Math. 122 (1900), 299-321.

[14] P. Schafheitlin, "Über die Gaussche u. Besselsche Differentialgleichung und eine neue Integralform der Letzteren", J. Reine Angewandte Math. 114 (1895), 31-44.

[15] T. I. Seidman, "Observation and prediction for one dimensional diffusion equations", J. Math. Anal. Appl. 51 (1975), 165-175.

[16] G. P. Tolstov, Fourier series (Prentice Hall, Englewood Cliffs, N.J., 1976).

[17] G. N. Watson, A treatise on the theory of Bessel functions (Cambridge University Press, 1944). 